\title{
Tracing and Its Observer Effect on Concurrency
}

\author{
Indigo Orton \\ indigo.orton@cl.cam.ac.uk \\ University of Cambridge \\ Department of Computer Science and Technology \\ Cambridge, UK
}

\author{
Alan Mycroft \\ alan.mycroft@cl.cam.ac.uk \\ University of Cambridge \\ Department of Computer Science and Technology \\ Cambridge, UK
}

\begin{abstract}
Execution tracing has an observer effect: the act of tracing perturbs program behaviour via its overhead, which can in turn affect the accuracy of subsequent dynamic analysis. We investigate this observer effect in the context of concurrent behaviour within JVM-based programs. Concurrent behaviour is especially fragile as task-scheduling ordering can change, which could even lead to deadlock via thread starvation under certain conditions. We analyse three dimensions of overhead, compute volume, memory volume, and uniformity, using a configurable-overhead tracer and a concurrency-performance analyser. We argue that uniformity is a key, and underappreciated, dimension of overhead that can have qualitative effects on program behaviour. Experimental results show that overhead significantly affects real-world concurrent behaviour and subsequent analysis, at times unintuitively.

CCS Concepts: - Software and its engineering $\rightarrow$ Software performance; Dynamic analysis; • Theory of computation $\rightarrow$ Concurrency; Program analysis; • General and reference $\rightarrow$ Performance; Measurement.
\end{abstract}

Keywords: Performance analysis and monitoring, Dynamic program analysis, Concurrency, Execution tracing

\section{ACM Reference Format:}

Indigo Orton and Alan Mycroft. 2021. Tracing and Its Observer Effect on Concurrency. In Proceedings of the 18th ACM SIGPLAN International Conference on Managed Programming Languages and Runtimes (MPLR '21), September 29-30, 2021, Münster, Germany. ACM, New York, NY, USA, 9 pages. https://doi.org/10.1145/3475738. 3480940

\section{Introduction}

Execution tracing is used to capture precise and fine-grained data about a program's behaviour. These data can be used to better understand and optimise program behaviour and

This work is licensed under a Creative Commons Attribution 4.0 International License.

MPLR '21, September 29-30, 2021, Münster, Germany

(C) 2021 Copyright held by the owner/author(s).

ACM ISBN 978-1-4503-8675-3/21/09.

https://doi.org/10.1145/3475738.3480940 to enable automatic program analysis. It is especially useful for performance analysis, which depends on data from real executions.

However, execution tracing naturally has an observer effect (also known as a probe effect); the act of tracing itself perturbs the behaviour being observed. This is caused by overhead incurred by tracing. The effect can be substantial enough that the recorded behaviour is significantly distorted from the untraced behaviour. Any analysis, manual or automatic, on sufficiently distorted data is inherently flawed and may lead to invalid conclusions.

There are many dimensions along which overhead can be evaluated, we consider three in particular:

Compute volume The amount of direct compute overhead (i.e. CPU cycles) introduced.

Memory volume The amount of memory consumed by the tracer. This indirectly incurs compute overhead by increasing page faults and can directly incur overhead if it consumes memory bandwidth in a bandwidth-constrained application.

Uniformity How uniformly overhead is incurred across threads (concurrent uniformity) and across time (temporal uniformity).

While all three inform the design of new execution tracers (such as which dimensions to prioritise for optimisation), they also elucidate the relative fragility of concurrent behaviour and analysis.

We argue that understanding the uniformity of overhead is essential to understanding its effect on program behaviour (Section 2). The common amortising mindset that "tracing adds $x \%$ overhead" is misguided as it does not account for the nuanced nature of overhead, instead assuming it is a consistent slow down of the program. Importantly, overhead can qualitatively affect a program's behaviour.

In this paper we investigate the effect of tracing-overhead on program concurrent behaviour - how and when a program uses concurrency - and execution duration in real-world Java programs. We also consider how distorted-behaviour in traces affects the accuracy and validity of subsequent concurrency-performance analysis. Various metrics are suitable for exploring the effect of the overhead dimensions above. We focus on four key metrics:

MTU Mean thread usage - effectively the integral of thread use over time, divided by total time. 
A-MTU Active threads MTU - active threads are those progressing computation at a given time.

W-MTU Waiting threads MTU - a thread is waiting when it is waiting on another thread to finish some work.

WCD Wall-clock duration of a program execution.

The key research questions addressed in this paper are:

1. How does the observer effect inherent in execution tracing affect program concurrent behaviour?

2. How do the various dimensions of tracing-overhead (compute, memory, and uniformity) differ in their effects?

3. How is subsequent concurrency-performance analysis affected by the observer effect?

To investigate these questions, we developed a low-overhead JVM tracer, Covetr (Configurable OVErhead TRacer), and augmented it to generate configured additional overhead (Section 3). To investigate the effect of tracing-overhead on subsequent program analysis we use a concurrency-performance analyser, Rehype, that we introduced in a previous paper [16] (summarised in Section 3.2).

Managed runtimes, and the JVM in particular, make it significantly easier to instrument and trace programs in a fast and safe way. Though native programs can be similarly instrumented, there is less available tooling and it is easier to introduce errors and inefficiencies. In particular, managed runtimes enable runtime instrumentation (e.g. during class loading), provide debug symbols (e.g. function signatures) by default, and (almost) automatically integrate inserted tracing code (e.g. JVM bytecode) into existing error handling and JIT compilation. Moreover, instrumentation tools can take advantage of greater runtime information (e.g. program state) to decide when and how to instrument and trace code.

Initial experimental results (Section 4) suggest that: the hypothesised observer effect does exist and can significantly affect concurrent behaviour; overhead dimensions vary in their effects; and increased overhead reduces analysis accuracy. While the results are specific to concurrent behaviour and concurrency-performance analysis, we suggest that the observer effect is broadly relevant to many forms of dynamic analysis.

The aim of this paper is to demonstrate the existence and relevance of the tracing observer effect on concurrent behaviour and analysis. We believe its relevance to dynamic analysis warrants further investigation. While the experimental evidence we present is a first step on this path of investigation, there is significant scope for further experimentation and analysis.

Finally, we position this paper among related work in Section 5 and conclude in Section 6.

\section{Uniformity in Tracing}

We wish to challenge an implied assumption in the statement "tracing adds $x \%$ overhead", the assumption is that such overhead does not significantly affect program behaviour, it merely slows the program (i.e. wall/user-clock duration) as though the processor, memory, and I/O speed had all been equally slowed. Tracing-overhead is not equivalent to a slower processor, it is not uniform as the overhead is only incurred when events are traced and events are not traced uniformly. Thus, even given uniform per-event overhead, the impact of tracing-overhead will be non-uniform.

Furthermore, as tracing-overhead fundamentally interacts with the broader system, by consuming limited resources, it will have a greater impact at points of resource contention and bottlenecks. Any such impact is further amplified as points of contention have the greatest opportunity for distorting program behaviour.

Thus, while the apparent outcome of tracing may be $x \%$ longer execution duration, the qualitative effect on behaviour may be greater. For example, task-scheduling may be affected, which could, in the worst case, lead to deadlock via thread starvation ${ }^{1}$. Similarly, increased overhead may cause a program to change modes of operation, e.g. an adaptive bitrate streaming system [19] choosing to degrade the quality of video segments. So, though $x \%$ might be acceptable in some instances (e.g. for non-performance-analysis applications, such as record/replay), it may not be so innocuous in other instances.

\section{Method}

In our experiments we use two systems: a configurableoverhead tracer and a concurrency-performance analyser.

\subsection{Configurable-Overhead Tracer}

Covetr is an execution tracer that takes in a trace-config and generates a trace-log. The trace-config specifies which functions of the target program should be traced. Covetr generates function and concurrency framework events (e.g. taskspawn events; other concurrency events, such as OS thread wait/wake, are not necessary as the task framework events provide sufficient information for our analysis). The vast majority of events generated are function-entry and -exit events. These contain a nanosecond time-stamp $\mathrm{p}^{2}$, a function identifier, and id for the thread that the event occurred on.

Covetr consists of two primary components, the instrumenter and the tracer. The instrumenter is essentially an aspect-oriented-programming tool that is configured by the trace-config. It intercepts class loading within the JVM and modifies class bytecode to insert tracing code. The tracer is an in-process utility that receives calls from tracing code and records corresponding events on disk as a trace-log.

\footnotetext{
${ }^{1}$ https://wiki.sei.cmu.edu/confluence/display/java/TPS01-J.+Do+not+ execute+interdependent+tasks+in+a+bounded+thread+pool

${ }^{2} \mathrm{We}$ assume the system clock is monotonic and consistent across threads (i.e. time-stamps can be compared). However, our method does not rely on this (time-stamps are not used for ordering).
} 
The tracer is designed for minimal overhead, prioritising low compute volume. To achieve this, it uses a client-server architecture with a single server and one client for each JVM thread. The clients generate logs of per-thread events and the server writes these to disk on a background thread. Logs are transferred using a bespoke lockless buffer exchange protocol.

Lockless buffer exchange. Each client uses two buffers, the active and inactive buffers. Each buffer has a boolean flag that indicates whether the client or the server has control of the buffer. The active buffer is always controlled by its containing client. Clients write new events to their active buffer. When a client's active buffer fills, the client attempts to swap its active and inactive buffers. If a client controls its inactive buffer, the buffers are swapped and the client sets the new inactive buffer to be controlled by the server. However, if a client's inactive buffer is controlled by the server when its active buffer fills, the client will expand its active buffer.

The server runs a periodic loop checking for client inactive buffers flagged as controlled by the server, when it finds one, it writes the buffer to disk. The server uses two further techniques to minimise occurrences of clients expanding their active buffers. First, the server can request specific clients swap their buffers before their active buffer reaches capacity. When a client generates an event, it checks its swaprequest flag, if set, the client immediately swaps its buffers. Second, the server has an additional exchange buffer which is given to a client in place of its inactive buffer when the server takes the inactive buffer. This enables clients to swap their buffers, even if the server is still writing their inactive buffer to disk.

Configurable overhead. For this work, we extend the traceconfig with an overhead-config specifying that additional synthetic overhead is to be incurred when recording an event basically it is easier to add tracing-overhead than to remove it! The overhead-config can specify both additional computation (simulated by hash operations on a sequence of random bytes) and additional memory allocation (malloc) by the tracing process, and also whether allocation has a corresponding free (allocating but not freeing memory enables isolating the effect of memory overhead from the allocation compute overhead). Ideally, we would like to be able to configure uniformity of tracing-overhead, however, as a uniform baseline is not possible (Section 2), we instead experiment with uniformity by adjusting which functions are instrumented (Section 4.1).

\subsection{Concurrency-Performance Analyser}

Rehype [16] takes a trace-log, generated by Covetr, and produces suggestions as to how the program's concurrency might be refactored to improve performance (such as inlining a task to reduce the amount of time threads in the program spend waiting). We call these suggestions changes and denote them using $\delta$, here treated as maps from tracelogs to trace-logs.

Given a trace-log $t$, Rehype produces a set of changes $\Delta$ and an estimated trace-log $\delta(t)$, for each change $\delta \in \Delta$. These approximate the trace-log the program would generate with $\delta$ implemented on the source code. Then, given a performance metric $\mu$ and writing the value of $\mu$ for $t$ as $\mu(t)$, Rehype calculates the estimated proportional improvement (EPI) of a change as:

$$
\operatorname{EPI}_{\mu}^{\delta}(t)=(\mu(\delta(t))-\mu(t)) / \mu(t)
$$

The experimental situation below is slightly more complex as we have a range of traces $t$ obtained by varying overheadconfig in the tracer.

\section{Experimental Results}

We perform experiments to investigate the three research questions (see Introduction). We define the various overhead configurations used in experiments in $\$ 4.1$, describe the experimental metrics in $\S 4.2$, report on the experiments in $\S 4.3$, and discuss experimental limitations in $§ 4.4$.

To investigate the real-world effects of tracing-overhead, we perform all experiments on an industrial Java API server, Acme. Acme uses a task-based concurrency model (centred on substantial usage of Java's standard thread pool interface, ExecutorService), has approximately 500k LoC, and has been in production for five years. Experiments execute a synthetic series-of-requests workload which simulates the standard use of Acme on a non-loaded machine. This provides a consistent workload to evaluate the effects of various overhead configurations.

All experiments were run on a Linux benchmarking machine with specifications: Intel Core i7-8700 CPU @ 3.20GHz, 32GB RAM, Clear Linux OS [1] 34630. Intel TurboBoost was disabled, the CPU scaling_governor parameter was set to performance, and hyper-threading was enabled as it provides a more realistic basis for concurrency analysis. Software: Java OpenJDK 1.8.0_292, Rust 1.50.0-nightly (Covetr is implemented in Rust). Experiments were run using Docker [13] containers ${ }^{3}$, Docker version 20.10.6. Max JVM heap size was $8.36 \mathrm{~GB}$.

\subsection{Configurations}

We use two baseline configurations:

base: instruments only concurrency constructs (such as thread-pool and task events) necessary to capture concurrent behaviour.

\footnotetext{
${ }^{3}$ Docker containers simplify benchmark environment management and consistency. On Linux, Docker containers have been shown to have negligible impact on CPU and memory speeds [2] (unlike virtual machines).
} 
std: standard, but unoptimised, configuration used by $R e-$ hype to identify changes for Acme. This configuration extends base by instrumenting all functions in Acme (i.e. the com. acme package, but not third-party library code).

We denote additional overhead configurations using their overhead type and volume (Section 3.1):

co-[x]: Extends std with compute overhead by performing $[x]$ hashes on each event; e.g. co-2 performs two hashes each event.

$\mathrm{db}-[\mathrm{x}]$ : Add compute overhead by performing hashes on each event, as co- $[x]$ does. However, unlike co- $[x], d b-[x]$ only instruments third-party library functions that are primarily called during concurrent phases of Acme (specifically, database access functions) instead of those instrumented by std. We use this to demonstrate the effect of overhead during phases with greater concurrency.

ma- $[\mathrm{x}]$ and $\mathrm{ml}-[\mathrm{x}]$ : Extends std with memory overhead by allocating $[x]$ bytes on each event; ma immediately frees the memory, while $\mathrm{ml}$ does not. These allow experiments to isolate memory use effects from the compute overhead of allocation (§3.1).

\subsection{Metrics}

We evaluate experiments on summary metrics of thread usage and wall-clock duration (WCD) as the overhead configuration is varied. We calculate the mean thread usage (MTU) across an execution for all occupied threads, active threads (A-MTU), and waiting threads (W-MTU). A thread is occupied when it is processing a task (as opposed to being idle in a thread pool); it is active when it is progressing computation; it is waiting when it is paused waiting for the result of another task. We derive additional metrics below to further evaluate the effects of overhead.

Metrics $\mu$ are naturally defined on trace-logs $t$. However, our experiments only vary configurations $c$ and not other inputs, hence it is convenient to regard $\mu$ also as functions of $c$. The same applies to EPI (§3.2).

Idea 1. We compare configurations $c$ using the value of a metric relative to the base configuration (i.e. $\mu(c) / \mu$ (base)). These derived metrics provide a direct measure of the distortion caused to concurrent behaviour by configurations' overhead. We define the relative effect (RE) of a metric $\mu$ as the absolute distance of the metric to the base version. That is, given a configuration $c$, the $\mu$-relative effect ( $\mu$-RE) is:

$$
\mu-\operatorname{RE}(c)=\left|\frac{\mu(c)}{\mu(\text { base })}-1\right|
$$

The ratio of MTU-RE to WCD-RE (shown in Fig. 2b) is useful as a measure of the proportional effect on concurrency and duration:

MTU-RE-to-WCD-RE-ratio $(c)=\operatorname{MTU}-\mathrm{RE}(c) / \mathrm{WCD}-\mathrm{RE}(c)$
Idea 2. To evaluate the effect of overhead on Rehype's analysis accuracy, we compare the EPI (Eq. 1) for changes that are identified by std (as base does not record enough data) and additional overhead configurations. Specifically, for a given configuration, we calculate the relative EPI (REPI) for each change both it and std identify and then take the mean REPI (MREPI) across all such changes. In short, MREPI diverging from $100 \%$ indicates overhead is affecting analysis accuracy (REPI represents the affect on accuracy for a single metric).

Assuming metric $\mu$, we write $\Delta^{c}$ for the changes identified by Rehype in a trace-log under overhead configuration $c$, and define $\Delta=\Delta^{c} \cap \Delta^{\text {std }}$. The REPI of change $\delta \in \Delta$ indicates the reduction in accuracy for that change caused by the configuration's overhead:

$$
\operatorname{REPI}_{\mu}^{\delta}(c)=\operatorname{EPI}_{\mu}^{\delta}(c) / \operatorname{EPI}_{\mu}^{\delta}(\mathrm{std})
$$

The MREPI is then the average reduction in accuracy caused by the overhead over common changes $\Delta$ :

$$
\operatorname{MREPI}_{\mu}(c)=\frac{1}{|\Delta|} \sum_{\delta \in \Delta} \operatorname{REPI}_{\mu}^{\delta}(c)
$$

Idea 3. Finally, the time-series metric of thread usage over a program execution (its concurrent profile) is useful to illustrate concurrent behaviour; its graphical form gives the shape of concurrency throughout an execution - i.e. when and how many threads are used. Two executions of the same program, with the same input and environment, should have almost identical concurrent profiles in the absence of overhead; Fig. 1b shows the concurrent profiles of three executions of Acme, under different overhead configurations.

\subsection{Experiments}

We devise separate experiments for each research question (see Introduction). The key takeaway is that: tracing-overhead can significantly distort program behaviour (to amplify or suppress concurrency), even when execution duration is largely unaffected.

RQ.1. How does the observer effect inherent in execution tracing affect program concurrent behaviour? To investigate this question we use the plainest form of overhead, compute volume, and correlate it with a change in MTU, reflecting a distortion to the program's concurrency. This correlation is illustrated in Fig. 1a (all raw values are provided in Table 1). The figure also shows that, for Acme, increased overhead decreases the MTU - making the program appear less concurrent. It is important to understand that while Acme does behave less concurrently (a greater proportion of the execution is spent on a sequential phase) given overhead, this is not reflective of its behaviour in a normal state without overhead (thus it merely appears less concurrent).

The intuition behind the reduction in apparent concurrency is that a particular sequential phase of execution within 


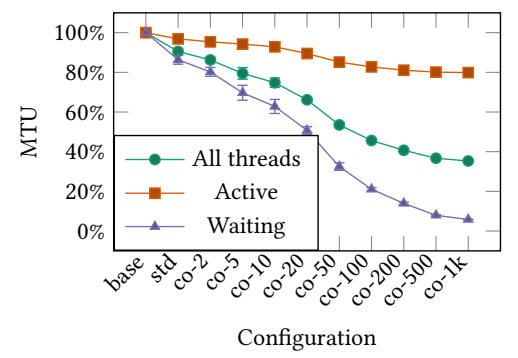

(a) Relative MTU for compute overhead configurations. Configurations approximately double overhead each increment.

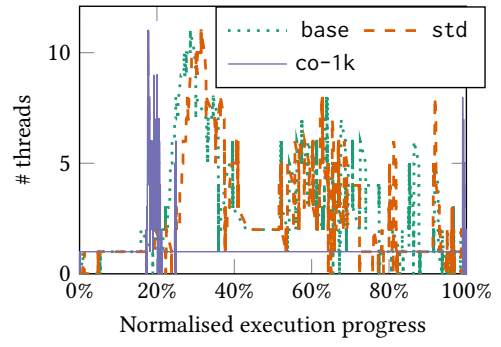

(b) Concurrent profiles (thread usage over normalised execution time) for the base, $\mathrm{std}$, and co- $1 \mathrm{k}$ configurations.

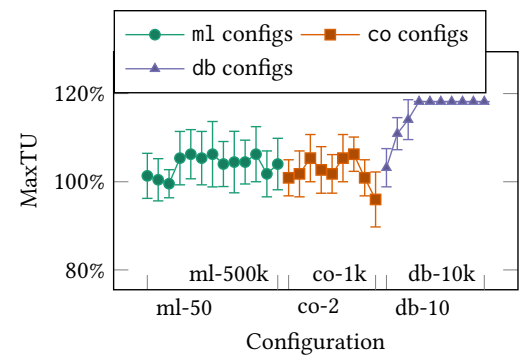

(c) Qualitative effect on program behaviour via MaxTU relative to base. A different MaxTU reflects a different task-scheduling.

Figure 1. The effect of compute overhead on program concurrent behaviour. All configurations were executed 20 times.

Acme generates more trace events and thus incurs more overhead. This is shown in Fig. $1 \mathrm{~b}$; the co-1k profile has an amplified sequential phase (approximately between $30 \%$ and $95 \%$ of the execution progress), while the base and std profiles appear more concurrent throughout. In those profiles, tracing incurs less overhead and so does not amplify the sequential phase as significantly.

Fig. 1a also illustrates how concurrency distortions can affect the apparent concurrent efficiency of a program. The W-MTU decreases more than the A-MTU, suggesting (if taken at face value) that the program is more efficiently using concurrency (as waiting threads are an inefficient use of resources [16]), than it does when executed without overhead.

Finally, Fig. 1c illustrates the change in maximum recorded concurrent thread use (MaxTU) due to overhead. A MaxTU change results from a task-scheduling change. This reflects a qualitative effect on program behaviour due to overhead. Note that the effect is not monotonic (though it appears so for the $\mathrm{db}-\star$ configurations due to the increased overhead on background threads), rather, qualitative effects can be chaotic.

RQ.1. Answer: the observer effect can significantly distort the concurrent behaviour of a program. Furthermore, overhead can have qualitative effects that are not monotonic.

RQ.2. How do the various dimensions of tracing-overhead (compute, memory, and uniformity) differ in their effects? We discuss this question in two parts: (i) the subtleties between compute and memory overhead; and (ii) uniformity and its fundamental nature. The difference between compute and memory overhead is primarily exhibited by the difference between MTU and WCD. The effect on MTU is bounded, while the effect on WCD is not.

Given a maximum recorded concurrent thread use MaxTU such that MaxTU > 1, the MTU is always less than it and greater than 1 (if the program does not deadlock), i.e. for a given configuration $c, 1<\operatorname{MTU}(c)<$ MaxTU. Moreover, the effect of increasing or decreasing overhead begins to level off as it nears these bounds. This effect is seen in Figs. 1a, 2a, and $2 \mathrm{c}$.

The WCD metric, however, has no such bound, it can continue to increase indefinitely (though it can never go negative). In fact, WCD-RE naturally accelerates as overhead increases and becomes the bottleneck in the program's execution, this is seen in Fig. $2 \mathrm{~d}$. The fact that co-* and $\mathrm{db}-\star$ configurations affect duration significantly is unsurprising as they directly add to the compute time. What is interesting, however, is that ml-* configurations do not show the same significant increase in WCD, despite it having levels of MTURE equivalent to the $d b-\star$ configurations. This difference is seen in the ratio of MTU-RE to WCD-RE (Fig. 2b).

We have argued that overhead uniformity cannot exist (Section 2). Yet, demonstrating the effect of uniformity in isolation would require a uniform baseline. A purelyuniform baseline cannot exist and a practically-uniform baseline cannot practically exist (practical uniformity could be constructed in a toy example, but not in a real-world system). As such, we must instead observe the effect of uniformity in other experiments, such as Fig. 1b. Here, the std and co-1k configurations track the same functions, however, the functions are not uniformly invoked across the execution. As co-1k incurs greater overhead, its profile shows that the functions are disproportionately invoked in the sequential phase.

Uniformity, as a dimension of overhead, is important to consider as it intrinsically affects the behavioural distortion caused by overhead. It is present in all of our experiments as it determines where overhead is incurred. While the experiments so far have depressed concurrency (relative MTU less than 100\%) the inverse distortion, an apparent increase in overall concurrency (relative MTU greater than 100\%), can also be observed. For example, Fig. 2c shows the MTU for $\mathrm{db}-\star$ configurations. These configurations increase the apparent concurrency by adding overhead to database access functions, which Acme primarily calls in background threads. 


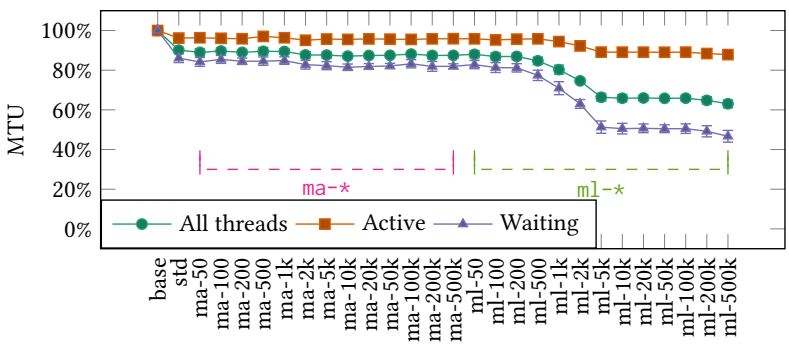

(a) Relative MTU for memory configurations.

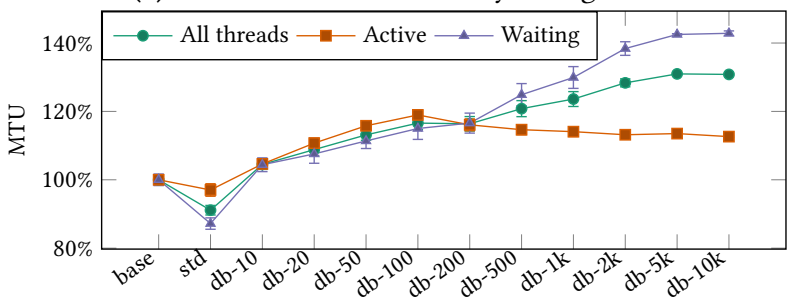

(c) Relative MTU for configurations that amplify concurrency.

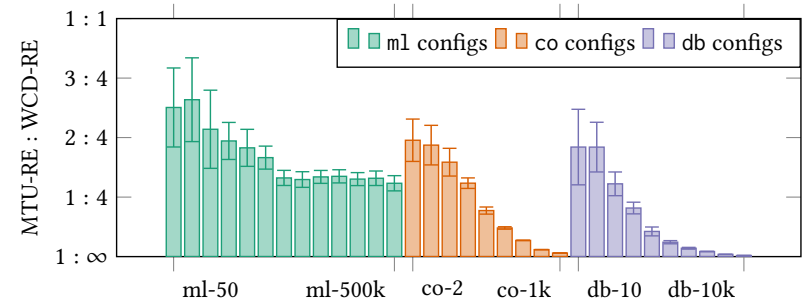

(b) Ratio of MTU-RE to WCD-RE (Eq. 3).

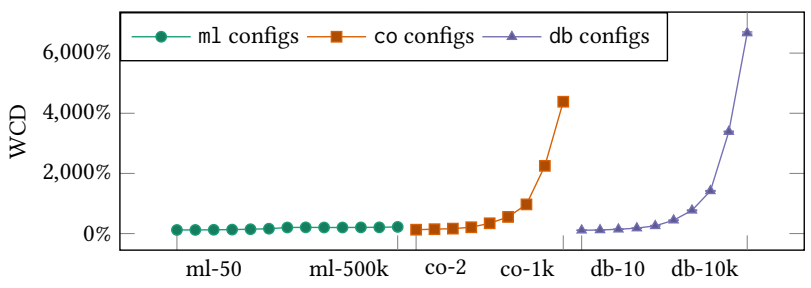

(d) Relative WCD for $\mathrm{ml}-\star$, $\mathrm{co}^{-*}$, and $\mathrm{db}-\star$ configurations.

Figure 2. The effect of various overhead configurations. All configurations were executed 20 times.

Whether distortion amplifies or depresses concurrency depends on which functions in the program generate the most trace events and when/where they are called (in concurrent or sequential phases). While this could, theoretically, result in uniformity if all threads produce identical events and overhead is perfectly uniform, such a scenario is unrealistic. In real programs, the number of events traced on each thread (concurrent uniformity) and at different times (temporal uniformity) will vary substantially causing overhead to be nonuniform (let alone naturally occurring non-uniformity due to tracing environment (e.g. cache behaviour)). Hence, in real programs, increased overhead correlates directly to increased concurrent behaviour distortion (i.e. increased MTU-RE).

RQ.2. Answer: increased compute and memory overhead correlates to increased MTU-RE and WCD-RE (though they are not proportional to each other), while uniformity controls the shape of the concurrent distortion. However, it is impractical to experimentally isolate overhead uniformity in real-world systems.

RQ.3. How is subsequent concurrency-performance analysis affected by the observer effect? Increased overhead reduces the accuracy of Rehype's estimations (Section 3.2). Fig. 3 illustrates this via two charts. The first (Fig. 3a) illustrates the reduced accuracy of estimations as the MREPI (Eq. 5) of multiple performance metrics (MTU, A-MTU, WMTU, and duration) for three $\mathrm{ml}-\star$ and co-* configurations ${ }^{4}$. The second (Fig. 3b) shows the percentage of changes identified for the std configuration that were identified by other configurations. The change in the set of identified changes

\footnotetext{
${ }^{4} \mathrm{The} \mathrm{db}-\star$ configurations are omitted as they do not instrument enough functions for Rehype to identify changes.
}

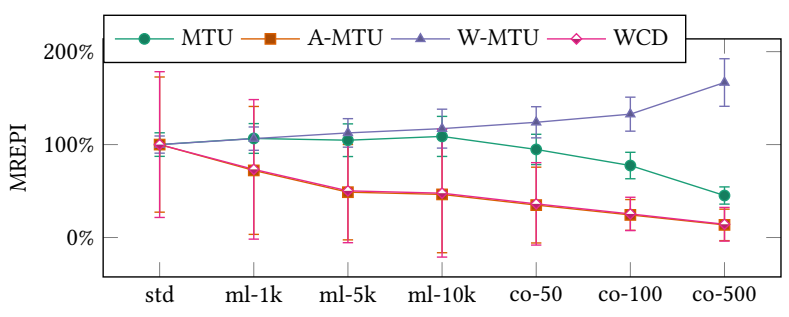

(a) The effect of overhead on Rehype's estimation accuracy (Eq. 5). Note that A-MTU and WCD overlap almost entirely.

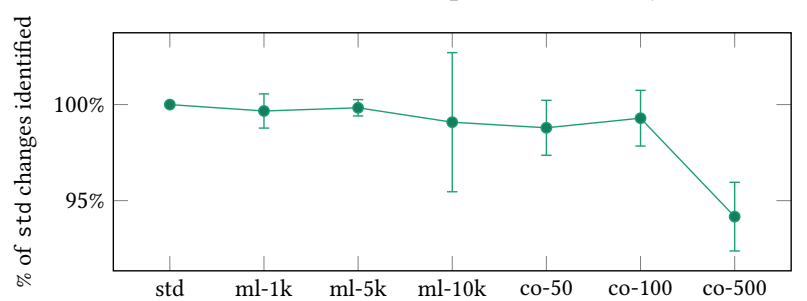

(b) The percentage of changes identified for the std configuration that are also identified for other configurations (i.e. $\left|\Delta^{c} \cap \Delta^{\mathrm{std}}\right| /\left|\Delta^{\mathrm{std}}\right|$ for a given configuration $c$ ).

Figure 3. The effect of overhead on Rehype's analysis. All configurations were executed 20 times.

for configurations with additional overhead represents a qualitative effect on the analysis. This shows that overhead can have a qualitative effect on concurrent behaviour (taskscheduling Fig. 1c) and, separately, a qualitative effect on subsequent analysis.

The effect of overhead is compounded in estimated tracelogs (§3.2), as they inherit both the behaviour distortions 
from the trace-log they are based off and the reduced accuracy of estimation due to overhead. The end result is an estimate of program behaviour, for a given change, that is far from the real behaviour the program would exhibit with the change implemented and zero tracing overhead. This results in developers having reduced (human) confidence in the validity of the estimations which can lead to reduced adoption of changes (even if they are, in fact, beneficial).

That being said, we find that, generally, Rehype is able to identify many changes as beneficial (in binary terms) despite the concurrency distortions. We hypothesise that this is due to the relative-improvement approach of Rehype's analysis.

RQ.3. Answer: analysis accuracy decreases as overhead increases, which matches intuition. This has been experimentally shown for Rehype and we suspect it holds for other analysis tools.

\subsection{Limitations}

These experimental results are limited to a single system Acme, running a single simulated workload, using four base metrics (MTU, A-MTU, W-MTU, and WCD), and analysed by Rehype. We argue that evaluation of tracer overhead to understand its various dimensions is important and that we have presented some initial results in these experiments. Future work should provide further rigour by evaluating more real-world systems, more program behaviour metrics, and more dynamic analysers.

\section{Related Work}

The observer effect has been previously studied in relation to performance analysis by Mytkowicz et al. [14] and synchronisation errors by Gait [3]. Mytkowicz et al. analysed how it can perturb performance data and system measurements so that a manual analysis (by a "performance analyst") may draw incorrect conclusions. Gait investigated how it modifies the frequency of occurrence of synchronisation errors in concurrent programs (inserting variable length delays affects when (and whether) synchronisation errors occur). Our work differs in that we specifically consider concurrent behaviour distortions and the effect on automatic concurrency-performance analysis.

More broadly we consider three areas of related work: various major strands of dynamic capture tools; tracing tools that treat overhead as a first-class problem; and research that investigates the effect of overhead in related domains.

Dynamic capture tools vary in the patterns and volumes of overhead incurred. At one end are profiling tools (e.g. perf [10] and Flight Recorder [8]), which perform lowoverhead periodic sampling to provide a rough snapshot of program performance behaviour; incurring small amounts of periodic overhead. At the other end are execution control systems (e.g. PIN [7]) that run programs in a managed environment and intercept $\mathrm{CPU}$ instructions; incurring large amounts of overhead constantly. In between there are: 1) kernel instrumentation tools (e.g. DTrace [5] and BPFs [4]) which insert probes in the Linux kernel to trace kernel events; 2) high-level tracing tools (e.g. Ruby Tracer [18]) which incur greater overhead to provide a simpler developer interface; and 3) dynamic instrumentation tools (e.g. Valgrind [15]) that incur high overhead (nullgrind, a minimal Valgrind configuration, has been reported [17] to incur more than $5 \mathrm{x}$ overhead on average) but provide a fine-grained tool for analysis of program behaviour, similar to execution control systems. In terms of overhead, Covetr exists between kernel instrumentation tools and high-level tracing tools, it traces more information than kernel tools, but is significantly more optimised than high-level tracers.

Mashtizadeh et al. [12] present Castor (a record/replay system) and Payer et al. [17] present memTrace (a memory tracing system), both are examples of systems that treat overhead as a first-class problem. Both leverage OS and hardware acceleration to achieve low overhead. For example, Payer et al. trace 32-bit code in 64-bit architectures, allowing memTrace to use an additional $2^{32}$ memory addresses beyond those that are used by the target program, as well as the extra registers available in x86_64 (over x86), meaning that the tracer and program operate on disjoint registers and memory regions. Castor, along with numerous hardware acceleration techniques, achieves low-overhead by being selective in what it records. Specifically, they only record sources of non-determinism (such as system calls). This is a key difference between Castor and Covetr; Covetr must record even deterministic events (e.g. function entry and exit) to capture enough performance information to accurately estimate the effect of changes and enough program information to identify sensible source-level changes.

Finally, the effect of various forms of overhead has been investigated in other fields. For example, the effect of cache overhead on allocation-heavy programs (Grunwald et al. [6]) and sorting algorithms (LaMarca and Ladner [9]), the effect of network overhead on cluster computing (Martin et al. [11]), and the impact of address translation on performance (Zhou et al. [20]).

\section{Conclusion}

Execution tracers have an observer effect: the act of capturing data perturbs the data captured. Tracing overhead has numerous dimensions, we considered three: compute, memory, and uniformity. Experimental results show tracingoverhead distorts the concurrent behaviour of a program, quantitatively and qualitatively, and can affect the accuracy and validity of subsequent analysis. Fundamentally, uniformity cannot exist in tracing and non-uniformity must be accepted, understood, and mitigated in execution tracers. 
Table 1. Experimental data. (All values are relative to the base configuration; " $90 \% \pm 1.60 \%$ " indicates a mean of 90 with a standard deviation of 1.60.)

Baseline configurations

co-* configurations

ma-* and $\mathrm{ml}$ - $^{*}$ configurations

\begin{tabular}{|c|c|c|c|c|}
\hline Config. & MTU & A-MTU & W-MTU & WCD \\
\hline base & $100.00 \% \pm 0.92 \%$ & $100.00 \% \pm 0.99 \%$ & $100.00 \% \pm 1.20 \%$ & $100.00 \% \pm 0.94 \%$ \\
\hline std & $90.53 \% \pm 1.60 \%$ & $96.87 \% \pm 1.38 \%$ & $86.34 \% \pm 2.24 \%$ & $114.16 \% \pm 3.67 \%$ \\
\hline co-2 & $86.26 \% \pm 1.39 \%$ & $95.37 \% \pm 1.28 \%$ & $80.22 \% \pm 2.25 \%$ & $129.05 \% \pm 6.02 \%$ \\
\hline co-5 & $79.48 \% \pm 2.92 \%$ & $94.24 \% \pm 1.97 \%$ & $69.70 \% \pm 3.76 \%$ & $145.36 \% \pm 11.12 \%$ \\
\hline co- 10 & $74.78 \% \pm 2.39 \%$ & $92.85 \% \pm 1.38 \%$ & $62.81 \% \pm 3.54 \%$ & $164.93 \% \pm 12.04 \%$ \\
\hline co- 20 & $66.17 \% \pm 1.12 \%$ & $89.49 \% \pm 0.70 \%$ & $50.73 \% \pm 1.87 \%$ & $210.15 \% \pm 7.78 \%$ \\
\hline co- 50 & $53.50 \% \pm 1.32 \%$ & $85.17 \% \pm 0.64 \%$ & $32.53 \% \pm 1.93 \%$ & $342.26 \% \pm 25.47 \%$ \\
\hline co- 100 & $45.64 \% \pm 0.46 \%$ & $82.74 \% \pm 0.41 \%$ & $21.08 \% \pm 0.78 \%$ & $552.55 \% \pm 21.35 \%$ \\
\hline co- -200 & $40.70 \% \pm 0.37 \%$ & $81.08 \% \pm 0.22 \%$ & $13.96 \% \pm 0.61 \%$ & $970.68 \% \pm 10.87 \%$ \\
\hline co-500 & $36.69 \% \pm 0.19 \%$ & $80.11 \% \pm 0.23 \%$ & $7.94 \% \pm 0.34 \%$ & $2251.81 \% \pm 9.67 \%$ \\
\hline co- $1 \mathrm{k}$ & $35.32 \% \pm 0.11 \%$ & $79.88 \% \pm 0.12 \%$ & $5.81 \% \pm 0.15 \%$ & $4384.03 \% \pm 10.16 \%$ \\
\hline ma-50 & $89.01 \% \pm 1.84 \%$ & $96.33 \% \pm 1.94 \%$ & $84.17 \% \pm 2.22 \%$ & $116.81 \% \pm 3.76 \%$ \\
\hline ma-100 & $89.68 \% \pm 1.53 \%$ & $96.06 \% \pm 1.31 \%$ & $85.45 \% \pm 2.00 \%$ & $116.17 \% \pm 2.95 \%$ \\
\hline ma-200 & $89.02 \% \pm 1.07 \%$ & $95.84 \% \pm 0.96 \%$ & $84.50 \% \pm 1.62 \%$ & $115.69 \% \pm 2.73 \%$ \\
\hline ma-500 & $89.53 \% \pm 1.92 \%$ & $97.04 \% \pm 2.10 \%$ & $84.57 \% \pm 2.10 \%$ & $117.00 \% \pm 4.28 \%$ \\
\hline ma-1k & $89.43 \% \pm 1.42 \%$ & $96.35 \% \pm 1.15 \%$ & $84.85 \% \pm 1.91 \%$ & $117.99 \% \pm 3.01 \%$ \\
\hline $\mathrm{ma}-2 \mathrm{k}$ & $87.69 \% \pm 1.61 \%$ & $95.10 \% \pm 1.66 \%$ & $82.80 \% \pm 2.22 \%$ & $122.57 \% \pm 3.84 \%$ \\
\hline ma-5k & $87.63 \% \pm 1.54 \%$ & $95.72 \% \pm 1.67 \%$ & $82.28 \% \pm 2.04 \%$ & $121.79 \% \pm 4.75 \%$ \\
\hline ma-10k & $87.08 \% \pm 1.17 \%$ & $95.54 \% \pm 1.63 \%$ & $81.49 \% \pm 1.62 \%$ & $121.21 \% \pm 3.86 \%$ \\
\hline ma-20k & $87.41 \% \pm 1.18 \%$ & $95.77 \% \pm 1.59 \%$ & $81.88 \% \pm 1.46 \%$ & $121.08 \% \pm 5.26 \%$ \\
\hline ma-50k & $87.50 \% \pm 0.96 \%$ & $95.64 \% \pm 1.29 \%$ & $82.12 \% \pm 1.31 \%$ & $122.41 \% \pm 5.23 \%$ \\
\hline $\mathrm{ma}-100 \mathrm{k}$ & $88.13 \% \pm 1.33 \%$ & $95.53 \% \pm 1.15 \%$ & $83.24 \% \pm 2.07 \%$ & $119.80 \% \pm 3.46 \%$ \\
\hline ma-200k & $87.43 \% \pm 1.63 \%$ & $95.80 \% \pm 1.50 \%$ & $81.90 \% \pm 2.47 \%$ & $121.01 \% \pm 5.03 \%$ \\
\hline ma-500k & $87.49 \% \pm 1.10 \%$ & $95.87 \% \pm 1.37 \%$ & $81.96 \% \pm 1.29 \%$ & $118.74 \% \pm 4.13 \%$ \\
\hline $\mathrm{ml}-50$ & $88.00 \% \pm 1.73 \%$ & $95.82 \% \pm 1.81 \%$ & $82.83 \% \pm 2.09 \%$ & $120.06 \% \pm 4.21 \%$ \\
\hline ml-100 & $86.87 \% \pm 1.85 \%$ & $95.26 \% \pm 1.49 \%$ & $81.33 \% \pm 2.48 \%$ & $121.09 \% \pm 4.83 \%$ \\
\hline $\mathrm{ml}-200$ & $86.94 \% \pm 1.65 \%$ & $95.65 \% \pm 2.17 \%$ & $81.18 \% \pm 2.10 \%$ & $126.00 \% \pm 5.58 \%$ \\
\hline $\mathrm{ml}-500$ & $84.73 \% \pm 1.88 \%$ & $95.81 \% \pm 1.50 \%$ & $77.41 \% \pm 2.61 \%$ & $132.06 \% \pm 5.67 \%$ \\
\hline $\mathrm{ml}-1 \mathrm{k}$ & $80.28 \% \pm 2.28 \%$ & $94.42 \% \pm 1.67 \%$ & $70.93 \% \pm 3.28 \%$ & $144.29 \% \pm 8.91 \%$ \\
\hline $\mathrm{ml}-2 \mathrm{k}$ & $74.63 \% \pm 1.25 \%$ & $92.21 \% \pm 1.42 \%$ & $63.00 \% \pm 2.23 \%$ & $161.66 \% \pm 6.11 \%$ \\
\hline $\mathrm{ml}-5 \mathrm{k}$ & $66.35 \% \pm 1.96 \%$ & $89.12 \% \pm 0.82 \%$ & $51.30 \% \pm 3.10 \%$ & $202.27 \% \pm 6.28 \%$ \\
\hline $\mathrm{ml}-10 \mathrm{k}$ & $65.88 \% \pm 1.84 \%$ & $89.10 \% \pm 1.09 \%$ & $50.53 \% \pm 2.69 \%$ & $206.75 \% \pm 15.52 \%$ \\
\hline $\mathrm{ml}-20 \mathrm{k}$ & $65.98 \% \pm 1.35 \%$ & $89.09 \% \pm 0.88 \%$ & $50.71 \% \pm 2.20 \%$ & $202.13 \% \pm 7.85 \%$ \\
\hline $\mathrm{ml}-50 \mathrm{k}$ & $65.83 \% \pm 1.19 \%$ & $89.05 \% \pm 0.86 \%$ & $50.48 \% \pm 1.97 \%$ & $201.92 \% \pm 8.20 \%$ \\
\hline $\mathrm{ml}-100 \mathrm{k}$ & $65.89 \% \pm 1.51 \%$ & $89.10 \% \pm 1.02 \%$ & $50.55 \% \pm 2.40 \%$ & $205.33 \% \pm 8.27 \%$ \\
\hline $\mathrm{ml}-200 \mathrm{k}$ & $64.80 \% \pm 1.84 \%$ & $88.40 \% \pm 0.83 \%$ & $49.20 \% \pm 2.82 \%$ & $207.58 \% \pm 8.52 \%$ \\
\hline $\mathrm{ml}-500 \mathrm{k}$ & $63.08 \% \pm 1.98 \%$ & $87.86 \% \pm 0.97 \%$ & $46.69 \% \pm 2.95 \%$ & $221.45 \% \pm 18.00 \%$ \\
\hline $\mathrm{db}-10$ & $104.49 \% \pm 1.45 \%$ & $104.62 \% \pm 1.12 \%$ & $104.40 \% \pm 2.01 \%$ & $109.90 \% \pm 1.12 \%$ \\
\hline $\mathrm{db}-20$ & $108.82 \% \pm 1.82 \%$ & $110.72 \% \pm 1.50 \%$ & $107.56 \% \pm 2.73 \%$ & $119.25 \% \pm 1.18 \%$ \\
\hline $\mathrm{db}-50$ & $113.13 \% \pm 1.56 \%$ & $115.79 \% \pm 1.10 \%$ & $111.36 \% \pm 2.22 \%$ & $143.31 \% \pm 2.98 \%$ \\
\hline $\mathrm{db}-100$ & $116.58 \% \pm 1.92 \%$ & $118.97 \% \pm 1.30 \%$ & $115.00 \% \pm 3.19 \%$ & $181.33 \% \pm 2.70 \%$ \\
\hline $\mathrm{db}-200$ & $116.38 \% \pm 2.12 \%$ & $116.07 \% \pm 1.75 \%$ & $116.58 \% \pm 2.92 \%$ & $256.41 \% \pm 11.48 \%$ \\
\hline $\mathrm{db}-500$ & $120.81 \% \pm 2.34 \%$ & $114.65 \% \pm 1.47 \%$ & $124.88 \% \pm 3.26 \%$ & $443.83 \% \pm 4.31 \%$ \\
\hline $\mathrm{db}-1 \mathrm{k}$ & $123.61 \% \pm 2.17 \%$ & $114.08 \% \pm 1.42 \%$ & $129.92 \% \pm 3.18 \%$ & $772.98 \% \pm 12.43 \%$ \\
\hline $\mathrm{db}-2 \mathrm{k}$ & $128.35 \% \pm 1.17 \%$ & $113.17 \% \pm 0.50 \%$ & $138.39 \% \pm 1.99 \%$ & $1418.88 \% \pm 11.30 \%$ \\
\hline $\mathrm{db}-5 \mathrm{k}$ & $130.97 \% \pm 0.20 \%$ & $113.52 \% \pm 0.23 \%$ & $142.51 \% \pm 0.22 \%$ & $3388.99 \% \pm 14.55 \%$ \\
\hline $\mathrm{db}-10 \mathrm{k}$ & $130.82 \% \pm 0.46 \%$ & $112.63 \% \pm 0.20 \%$ & $142.85 \% \pm 0.70 \%$ & $6667.10 \% \pm 37.46 \%$ \\
\hline
\end{tabular}


Furthermore, we have argued that uniformity is an essential, and understudied, dimension of overhead that warrants further investigation.

In future work we plan to evaluate tracing-overhead on more real-world programs and against more metrics. We also plan to investigate how tracing-overhead can qualitatively affect application functionality, such as in adaptive video streaming where overhead may cause video quality negotiation to have a different outcome.

\section{Acknowledgments}

We thank Lucian Carata for his many helpful suggestions and feedback; we also thank the anonymous referees. The first author was funded by the Engineering and Physical Sciences Research Council (EPSRC), the Cambridge Trusts, and the University of Cambridge Department of Computer Science and Technology.

\section{References}

[1] Clear Linux 2021. Clear Linux* Project. https://clearlinux.org/. Accessed May 2021.

[2] Wes Felter, Alexandre Ferreira, Ram Rajamony, and Juan Rubio. 2015. An updated performance comparison of virtual machines and Linux containers. In 2015 IEEE International Symposium on Performance Analysis of Systems and Software (ISPASS). 171-172. https://doi.org/10. 1109/ISPASS.2015.7095802

[3] Jason Gait. 1986. A probe effect in concurrent programs. Software: Practice and Experience 16, 3 (1986), 225-233. https://doi.org/10.1002/ spe.4380160304

[4] Brendan Gregg. 2019. BPF Performance Tools: Linux System and Application Observability (1st ed.). Addison-Wesley Professional.

[5] Brendan Gregg and Jim Mauro. 2011. DTrace: Dynamic Tracing in Oracle Solaris, Mac OS X and FreeBSD (1st ed.). Prentice Hall Press, USA.

[6] Dirk Grunwald, Benjamin Zorn, and Robert Henderson. 1993. Improving the Cache Locality of Memory Allocation. In Proceedings of the ACM SIGPLAN 1993 Conference on Programming Language Design and Implementation (Albuquerque, New Mexico, USA) (PLDI '93). Association for Computing Machinery, New York, NY, USA, 177-186. https://doi.org/10.1145/155090.155107

[7] Intel PIN 2021. Pin - A Dynamic Binary Instrumentation Tool. https://software.intel.com/content/www/us/en/develop/articles/ pin-a-dynamic-binary-instrumentation-tool.html. Accessed june
2021.

[8] Java Flight Recorder 2021. Java Flight Recorder. https: //docs.oracle.com/javacomponents/jmc-5-4/jfr-runtimeguide/about.htm\#JFRUH170.

[9] Anthony LaMarca and Richard E Ladner. 1999. The Influence of Caches on the Performance of Sorting. Fournal of Algorithms 31, 1 (1999), 66104. https://doi.org/10.1006/jagm.1998.0985

[10] Linux perf 2021. perf: Linux profiling with performance counters. https://perf.wiki.kernel.org/index.php/Main_Page.

[11] Richard P. Martin, Amin M. Vahdat, David E. Culler, and Thomas E. Anderson. 1997. Effects of Communication Latency, Overhead, and Bandwidth in a Cluster Architecture. SIGARCH Comput. Archit. News 25, 2 (May 1997), 85-97. https://doi.org/10.1145/384286.264146

[12] Ali José Mashtizadeh, Tal Garfinkel, David Terei, David Mazieres, and Mendel Rosenblum. 2017. Towards Practical Default-On Multi-Core Record/Replay. SIGPLAN Not. 52, 4 (April 2017), 693-708. https: //doi.org/10.1145/3093336.3037751

[13] Dirk Merkel. 2014. Docker: lightweight linux containers for consistent development and deployment. Linux journal 2014, 239 (2014), 2.

[14] Todd Mytkowicz, Peter F Sweeney, Matthias Hauswirth, and Amer Diwan. 2008. Observer effect and measurement bias in performance analysis. Computer Science Technical Reports CU-CS-1042-08, University of Colorado, Boulder (2008).

[15] Nicholas Nethercote and Julian Seward. 2007. Valgrind: a framework for heavyweight dynamic binary instrumentation. ACM SIGPLAN Notices 42, 6 (June 2007), 89-100. https://doi.org/10.1145/1250734. 1250746

[16] Indigo Orton and Alan Mycroft. 2021. Refactoring Traces to Identify Concurrency Improvements. In Proceedings of the 23rd ACM International Workshop on Formal Techniques for Java-like Programs (FTffP '21) (Virtual, Denmark). Association for Computing Machinery, 8. https://doi.org/10.1145/3464971.3468420

[17] Mathias Payer, Enrico Kravina, and Thomas R. Gross. 2013. Lightweight Memory Tracing. In 2013 USENIX Annual Technical Conference (USENIX ATC 13). USENIX Association, San Jose, CA, 115126. https://www.usenix.org/conference/atc13/technical-sessions/ presentation/payer

[18] Ruby Tracer 2021. Ruby/Tracer. https://github.com/ruby/tracer. Last accessed June 2021.

[19] Kevin Spiteri, Rahul Urgaonkar, and Ramesh K. Sitaraman. 2020. BOLA: Near-Optimal Bitrate Adaptation for Online Videos. IEEE/ACM Transactions on Networking 28, 4 (2020), 1698-1711. https://doi.org/10.1109/ TNET.2020.2996964

[20] Yufeng Zhou, Xiaowan Dong, Alan L. Cox, and Sandhya Dwarkadas. 2019. On the Impact of Instruction Address Translation Overhead. In 2019 IEEE International Symposium on Performance Analysis of Systems and Software (ISPASS). 106-116. https://doi.org/10.1109/ISPASS.2019. 00018 